\title{
Abrupt Deterioration of COVID-19 Patients and Spreading of SARS COV-2 Virions in the Lungs
}

\author{
N. Filipovic @ ${ }^{1,2}$ I. Saveljic, ${ }^{1,2}$ K. Hamada, ${ }^{3}$ and A. Tsuda ${ }^{4}$ \\ ${ }^{1}$ Faculty of Engineering, University of Kragujevac, Sestre Janjic 6, 34000 Kragujevac, Serbia; ${ }^{2}$ Biomedical Research and \\ Development Center for Bioengineering (BIOIRC), Prvoslava Stojanovica 6, 34000 Kragujevac, Serbia; ${ }^{3}$ Department \\ Respiratory Medicine, Nara Medical University, Shijo 840, Kashihara, Nara 634-8521, Japan; and ${ }^{4}$ Tsuda Lung Research, \\ Shrewsbury, MA 01545, USA
}

(Received 5 October 2020; accepted 21 October 2020; published online 2 November 2020)

Associate Editor Stefan Duma oversaw the review of this article.

\begin{abstract}
A unique feature of COVID-19 interstitial pneumonia is an abrupt progression to respiratory failure. Our calculation shows that this abrupt deteriorate may be caused by a sudden shift in the spread of virus-laden bioaerosols through the airways to many different regions of the lungs from the initial site of infection.
\end{abstract}

On the CDC website" it says "Clinicians should be aware of the potential for some patients (of COVID-19) to rapidly deteriorate one week after illness onset". The concept of "cytokine storm"4 is discussed to explain this rapid deterioration. But why does this happen several days after the initial infection? While many mechanisms possibly contribute to spread of the infection, such as lymphatic circulation, we hypothesize that a major driver of the abrupt deterioration several days after the initial infection is caused by a sudden shift in the spread of virus-laden bioaerosols through the airways to many different regions of the lungs from the initial site of infection. Clinically, severe SARS COV-2 infections exhibit multi-region patchy patterns of ground-glass opacity (GGO) throughout the lungs detected by computed tomography $(\mathrm{CT}){ }^{3}$ This CT presentation is a unique characteristic of COVID-19 and is consistent with our hypothesis.

Address correspondence to N. Filipovic, Faculty of Engineering, University of Kragujevac, Sestre Janjic 6, 34000 Kragujevac, Serbia. Electronic mail: fica@kg.ac.rs
To explore this possibility, we have made a computer simulation. Suppose some part of the lung is initially infected (Figure 1a). Pulmonary edema develops locally and a meniscus with virus-laden fluid may be formed. During breathing, the meniscus is stretched and can rupture, releasing a number of small droplets called bioaerosols. ${ }^{2}$ The bioaerosols move toward the airway opening with the expired air; while most of the bioaerosols exit the body, some of them remain suspended in the airways. In the next inhalation, some of the exited particles may reenter the lungs with little deposition due to the low level of diffusion. The particles, which remain in the lung, are likely to retrace their path back to the original position (Figure $1 b)$.

As the disease progresses, however, the distribution of airflow in the lungs changes. The infected part of the lungs contributes to a decreased volume of airflow, altering the airflow dynamics, and, at the same time, an increased production of virus-laden bioaerosols. This shift of airflow pattern distributes virus-laden bioaersols to other, even distant, parts of the lungs (Figure 1c). If multiple areas of the lungs are simultaneously infected, an overwhelming innate immune response may occur resulting in a widely-distributed "cytokine storm". Considering the negligible (but non-zero) diffusivity of bioaerosols, the observation that the deterioration occurs several days after the initial infection. Our hypothesis suggests that targeting therapies to slow primary GGO lesion growth and to control airflow patterns could slow intra-lung viral spreading and help prevent the rapid respiratory deterioration observed in severe COVID-19 patients. 

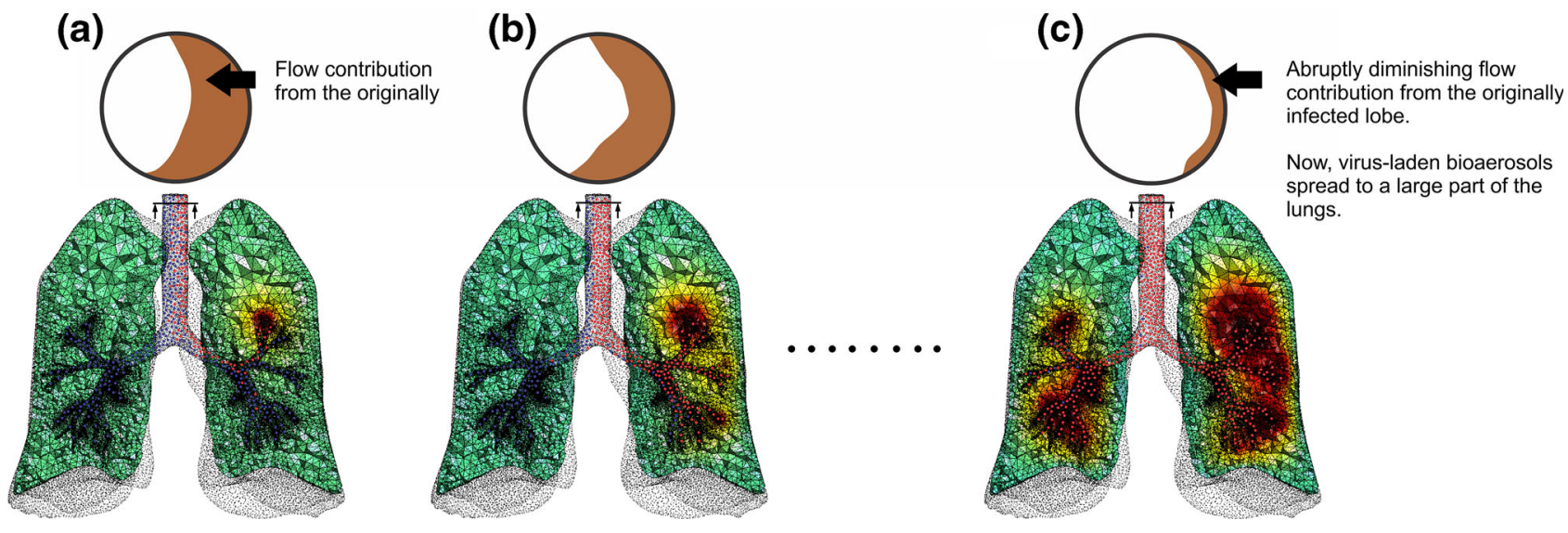

FIGURE 1. SARS COV-2 virus spreading in the lungs (see also a video animation in supplementary material). Flow distribution in the lungs is mapped on the cross-section of the trachea shown at the top of each figure. (a) Suppose some part of the lung is initially infected by inhaled SARS COV-2 virus (in this example, we assume that the left upper lobe is initially infected). As a result, near the lesion, virus-laden meniscus may be formed and a rupture of those menisci may form a number of virus-laden small bioaerosols (shown as red dots). (b) Those bioaerosols move toward the airway opening with the expired air. While most of them exit from the body, some of them may remain suspended in the airways. Some of particles, which exit the body, may reenter the lungs in the next inhalation and move around the lungs, but deposition of those reentered particles may be minuscule due to their low diffusivity. Some of the particles, which remain suspended in the airways retrace back to their original position due to the negligible (but no zero) diffusivity. (c) However, as the disease progresses, the production of virus-laden bioaerosols increases, the tissues available for gas exchange become smaller and smaller as the infected lesion becomes larger and larger. This may dramatically alter the distribution of airflow in the lungs, resulting in a substantial spread the infection to other parts of the lungs. This may cause cytokine storm. A dramatic change of flow distribution in the lungs can be seen as a significant alteration cross sectional map pattern from Figs. 1a, to 1c.

\section{FUNDING}

None.

\section{CONFLICT OF INTEREST}

All authors state no financial associations with this work.

\section{ELECTRONIC SUPPLEMENTARY MATERIAL}

The online version of this article (https://doi.org/10. 1007/s10439-020-02676-w) contains supplementary material, which is available to authorized users.

\section{AUTHOR CONTRIBUTIONS}

AT: designed the study. NF and IS: performed the numerical simulation. NF, IS and AT: designed and produced figures and videos. NF, IS, KH, and AT: reviewed, analyzed, and interpreted the data. AT: wrote the note. All authors discussed the results and commented on the letter.

\section{REFERENCES}

${ }^{1}$ CDC Coronavirus Disease 2019 (COVID-19). https://ww w.cdc.gov/coronavirus/2019-ncov/hcp/clinical-guidance-ma nagement-patients.html.

${ }^{2}$ Malashenko, A., A. Tsuda, and S. Haber. Propagation and breakup of liquid menisci and aerosol generation in small airways. J. Aerosol. Med. Pulm. Drug Deliv. 22(4):341-353, 2009.

${ }^{3}$ Salehi, S., A. Abedi, S. Balakrishnan, and A. Gholamrezanezhad. Coronavirus Disease 2019 (COVID-19): a systematic review of imaging findings in 919 patients. AJR 215:1-7, 2020. https://doi.org/10.2214/AJR.20.23034.

${ }^{4}$ Tisoncik, J. R., M. J. Korth, P. Cameron, C. P. Simmons, J. Farrar, T. R. Martin, and M. G. Katzea. Into the eye of the cytokine storm. Microbiol. Mol. Biol.Rev. 76(1):16-32, 2012.

Publisher's Note Springer Nature remains neutral with regard to jurisdictional claims in published maps and institutional affiliations. 